\title{
Massive Open Online Courses (MOOCs) in The United States, China, and India
}

\author{
Li, Yanxuan ${ }^{1, a}$ \\ ${ }^{1}$ Simon Business School, University of Rochester, Rochester NY 14627, United States \\ aYanxuan.K.Li@gmail.com
}

Keywords: MOOCs, Massive Open Online Courses, United States, China, India, Higher Education.

\begin{abstract}
MOOCs is considered a disruptive concept in world's education industry today. "Borderless access," "free of cost," and "unlimited enrollment" are the core attributes of MOOCs. Ideally, these characteristics allow students with various backgrounds to access quality higher education from any place in the world. In reality, there are barriers exist to limit MOOC access to particular groups of people in different geopolitical or socioeconomic society. This paper looks into the detail characteristics of MOOCs in three distinct countries: The United States, China, and India. From the comparison, we found that different cultural background, social needs, and economic development status will shape the MOOC industry, participants' characteristics, and industry development differently across countries.
\end{abstract}

\section{Introduction}

The concept of Massive Open Online Courses (MOOCs) was first introduced in 2010 by David Cormier [1]. The name of the concept speaks for itself. It refers to the online courses characterized by universal participation, cost-free, and admission criteria-free. It is usually considered as an important step to broaden access to higher education on a global level. First MOOC was offered by Stanford professors in late 2011, enrollment quickly increased to 160,000 students worldwide. Starting from the United States, this disruptive concept has taken higher education by storm. The New York Times later defined 2012 as "The year of the MOOC," because many MOOC providers emerged in 2012 and cooperated with top universities to provide quality courses [2]. Top universities offering MOOCs include Stanford, Harvard, University of Pennsylvania, MIT, Princeton University, and the University of Michigan.

MOOCs have estimated 58 million students now, and in 2016 alone, around 23 million people joined in the MOOC family for the first time. The top five MOOC providers today in the world are Coursera, edX, XuetangX, FutureLearn, and Udacity. As shown in Figure 1, the total number of MOOCs increase to 6,850 from over 700 universities in 2016 [3].

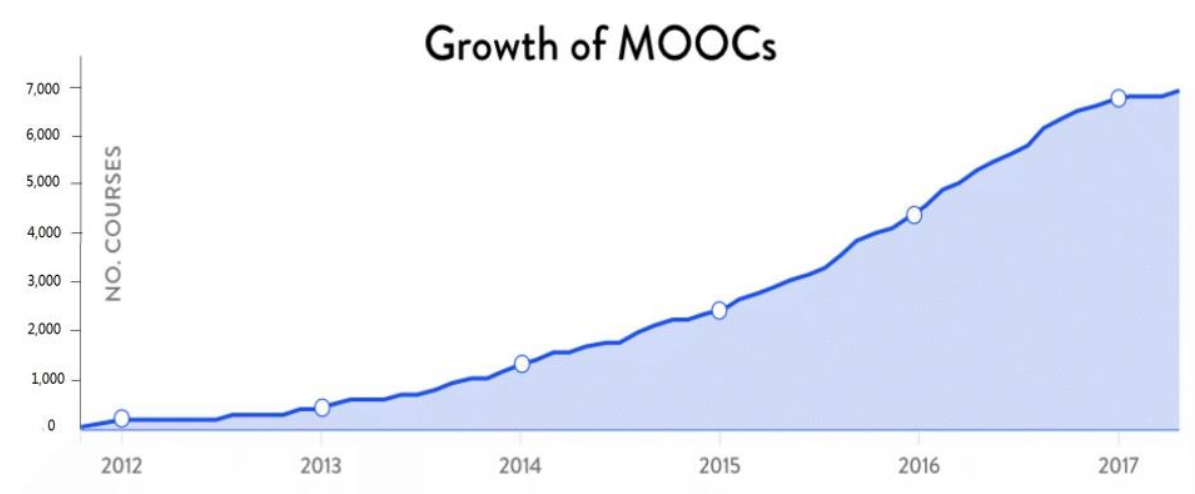

Fig. 1 Growth of MOOCs. (This figure is reproduced from Ref. 3)

Although MOOC is valued as a feasible solution for the expanding demand for higher education in the world, and a remedy for the unbalanced educational opportunity distribution. Moreover, the 
design of MOOCs as having no enrollment criteria and charging almost no cost to students should allow the online classrooms to have a more diverse population. Many studies still suggest this is not the case for current MOOC system:

"Despite the optimistic and aspirational declarations of many MOOC providers, these courses are not, as of yet, making education "borderless, gender-blind, race-blind, class-blind, and bank account-blind" [4].

Indeed, studies show that a vast majority of the MOOC students are from English speaking country and population [5], the majority of MOOC students are already well educated across countries [4], and the marginalized group in a particular society becomes even more underrepresented in MOOC classroom [6,7]. In addition to that, The MOOC industry exhibits different developing patterns in various society. Many factors shape the MOOC industry in different societies, including language, cultural background, economic status, and many others. It is necessary to recognize the key elements to better serves students in a similar background.

As of today, The United States is still by far the country with most MOOC students, which is $36.2 \%$ of all MOOC students worldwide. Followed by India at $13 \%$, and China at $9.5 \%$ [8]. Though different in order, the top three most populous country in the world is also China, India, and the U.S. [9]. Not only because they are the biggest MOOC market, but these three countries are different enough to represent individual cultural background and socioeconomic status. In this paper, we will examine the MOOC industry in these three countries from various perspectives: MOOC platform, participants characteristics, motivations, behaviors, and industry developments.

\section{Difference in MOOC Platform and Course Selection}

MOOC providers are platforms that facilitate various online courses, listing documents like video lectures, study materials, problem sets, and even users forums. The video lecture is usually the primary source of instruction, supported by additional readings and other assignments. At the end of a course, many platforms will offer a certified exam to test the effectiveness of student's study.

Coursera is currently the largest MOOC provider in the world with over 23 million students [3]. It partnered with 150 top universities in 29 countries today. It offers more than 2,184 courses, and around 700 of these courses were added in 2016. Coursera has a unique program called "Specialization," where each specialization contains a sequence of classes to teach a particular skill. The most popular specializations these days are Data Science, Python for Everybody, Excel to MySQL, and Business Foundations [10].

With over six million users, XuetangX is the third biggest MOOC providers worldwide. It is the only non-English MOOC platform of the top five providers in the world. XuetangX is based in Beijing, China, initiated by Tsinghua University, and it now has about 400 courses, a majority of them are in Chinese. In catering to the needs of Chinese MOOC students, XuetangX developed terminal applications for Android, iOS, IPad, and smart TV [11].

According to Class Central, the \#1 search engine for online courses, $19.3 \%$ of all MOOCs are in business and management subject, followed by computer science and programming at $17.4 \%$. Subjects like science, social sciences, humanities, and education have a similar share at $10.4 \%$, $9.82 \%, 9.82 \%$, and $9.26 \%$, respectively [3]. Not surprisingly, applied skills in the field of business and technology are in popular demand.

In the United States, $44.6 \%$ of the MOOC students enrolled in science and healthcare-related classes. In 2016, 49.31\% of the Chinese MOOC students learned a foreign language (mostly English) and $45.97 \%$ studied information technology [12]. Indian MOOC students prefer highly technical classes. The most popular courses in India is "Programming Mobile Applications for Android Handheld Systems." Followed by two Python programming classes and machine learning classes [13]. Another study suggests that U.S. students are less likely to take classes to enhance their skills for a job or their education compared to their non-U.S. counterparts [4]. Apparently, students from different countries have their patterns in choosing MOOCs. We will examine the reasons behind these differences in later sections. 


\section{Distinct Participant Demographic Patterns}

Within the United States, MOOC students are older comparing to MOOC students from China and India. In a survey of 32 University of Pennsylvania MOOCs with 11,933 United States respondents, only $23.5 \%$ of US MOOC students are under 30, significantly lower than the average at $41.1 \%$. Also, the percentage of retired MOOC students is at $14 \%$ in the U.S., comparing to $6.8 \%$ on average. Looking at employment status, only $9.8 \%$ of the U.S. respondents are currently students in traditional school. $69.5 \%$ of the MOOC students are employed. While the U.S. already has the highest percentage of the general population with a college degree at $31 \%$, the percentage of MOOC student population with a college degree is also high at $87 \%$. Regarding gender, $48.1 \%$ of the U.S. MOOC students are male, and $49.4 \%$ are female, while $56.9 \%$ of the global MOOC students are male and only $41.3 \%$ are female. In the U.S., no significant gender difference was shown [4]. For ethnicity, $77.03 \%$ of the U.S. MOOC participants identify themselves as white, but only $72.4 \%$ of the population in the U.S. identify themselves as white $[6,14]$, showing that white MOOC student is overrepresented compared to the U.S. population.

In China, MOOC students are younger. Based on a report on 30,187 Chinese MOOC students, $69.32 \%$ of them are in the age group of $17-26$. In a major contrast to the U.S. data, $50.52 \%$ of the respondents are students in traditional school, and only $48.16 \%$ are employed. However, the report also addresses that the percentage of working professionals in MOOCs are increasing every year. The percentage of the general population with a college degree is $15.35 \%$ [15], but the percentage of MOOC student population with a college degree is $92.18 \%$. Similar to other countries, the majority of the MOOC students are already the academic elites in their society. In China, $47.38 \%$ of the MOOC students are male, and $52.18 \%$ are female, which is a $4.8 \%$ gap between the number of male and female MOOC students [12].

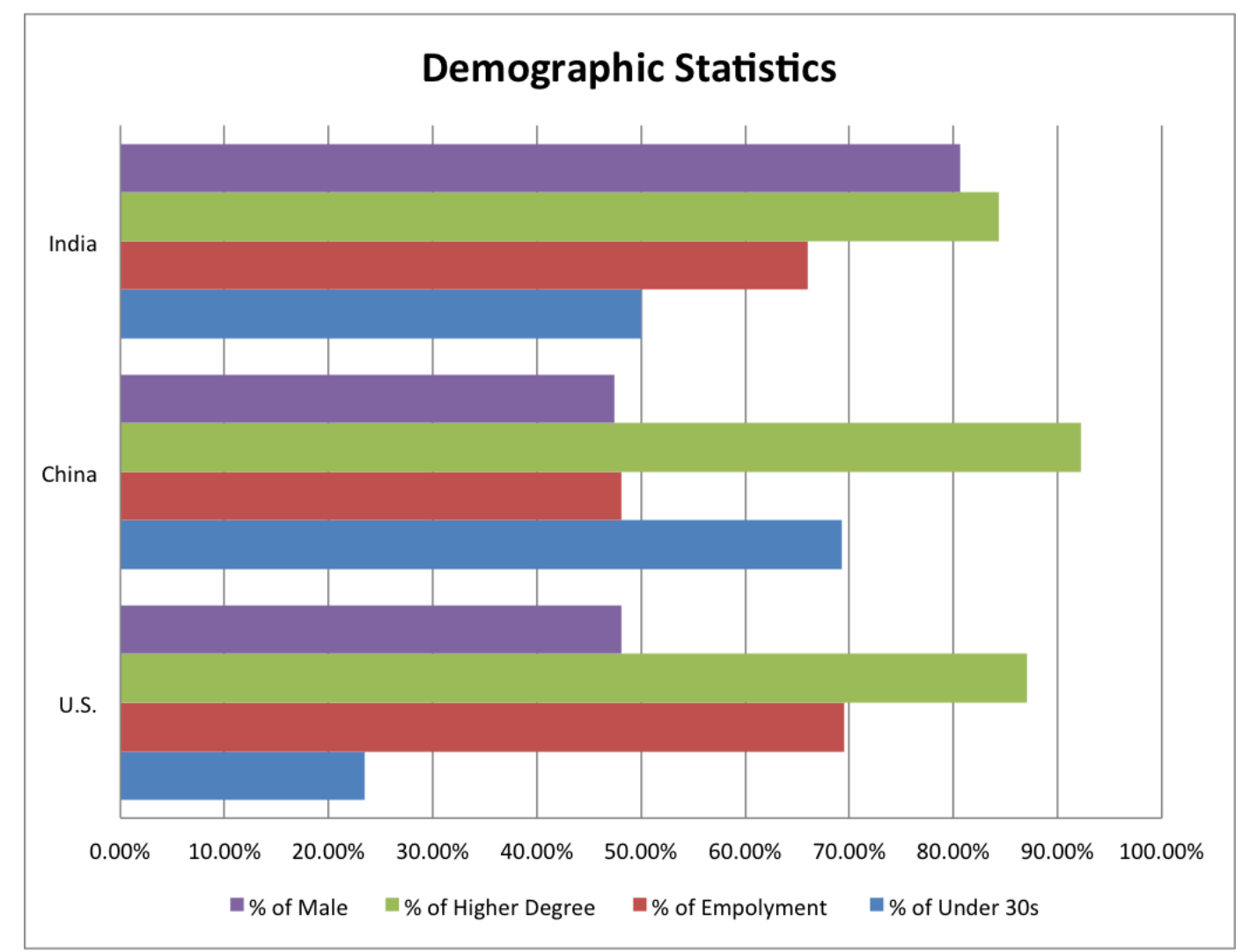

Fig. 2. MOOC demographic statistics for India, China, and the U.S. Purple, the percentage of male; green, the percentage of higher degree; red, the percentage of employment; blue, the percentage of under the $30 \mathrm{~s}$.

For India, the median age of Indian MOOC students is $26.32 .2 \%$ of the respondents are full-time students. Similar to the United States, employed professionals are $66 \%$ of all respondents. 
Regarding educational attainment, the percentage of Indian MOOC students with a college degree is at $84.3 \%$, while the percentage of the general population with a college degree is only $8.15 \%$ [16]. Although metrics like age, employment status, and educational attainment are in line with the United States or China or both, the gender breakdown of Indian MOOC students is in stark contrast with both countries: $80.6 \%$ of the MOOC students are male, and only $19 \%$ of them are female [7]. The Gender Parity Index (GPI) indicates that India's educational attainment score on gender parity is only at the 113th place out of 144 countries. The current higher education enrollment statistics show $44.2 \%$ of all students are female [17]. Although still under representative, it is a lot higher than the female MOOC enrollment rate in India. Alcorn identified many reasons for this gender disparity in MOOCs, "unequal access to technology, lower rates of female participation in STEM fields, and limited job prospects for females" [7].

\section{Varying Purposes and Motivations}

As shown in the previous section, significant demographic differences do exist in all three countries. The root of these differences is related to social class, social economic, and cultural reasons. It is easy to imagine that each group of MOOC students also have their unique purpose and motivation when studying. Several studies reveal a wide variety of motivations for taking MOOCs, including but not limited to: employment opportunities, the knowledge and skills gained, the entertainment value of the course, supplement current offline study $[4,12,18]$. However, a few research has segmented the respondent by country and connected the characteristics of MOOC students in different countries to their specific motivation of studying. In a MOOCs survey on students from over ten different countries, 50.05\% of the MOOC students reported "Curiosity, just for fun" as their intention of participating in MOOC. Meanwhile, $43.9 \%$ also acknowledge that "Gain specific skills to do my job better" as their reason for studying [4].

For the U.S. respondents specifically, 55.5\% take MOOCs for curiosity or just for fun, which is about 5\% higher than the global average. Another study also suggests that U.S. MOOCs participants were not motivated by educational advancement in general. $70.34 \%$ the U.S. MOOC participants were "not at all" motivated by educational achievement [6]. This phenomenon shows that in the U.S., "MOOC culture is mostly a 'free' culture" [19]. Comparing to China and India, students from the United States tend to have the least "opportunity cost" in taking MOOCs. Many of them are already highly educated, have an established career, and study MOOCs only for the purpose of learning. In China, $55.61 \%$ of the students take MOOCs for interest. But at the same time, 51.50\% still states that they take MOOCs to advance in their professional skill [12]. These students are making "investment" on their time to study materials that they believe will help them on their future career path. Although no survey data is available in India, Alcorn suggests that the primary objective of Indian MOOC students is to develop skills that help them at their current or future job, followed by the idea of supplementing traditional higher education [7].

In theory, MOOCs is considered the most recent effort to "broaden access to higher education and postsecondary coursework/knowledge by removing many of the institutional barriers" [6]. However, this is not happening in the U.S., China, and India at least. The majority of the MOOC students from these countries already have a college degree, and their reasons for enrolling in MOOCs are either because of personal interest and curiosity or because of professional development needs. Who and where are the educational seekers in these countries? A research found MOOC students in developing countries and lower socioeconomic status are more likely to be education seekers [20]. This being said, India and China, theoretically, are more likely to have MOOC students seeking educational benefits. Why are we not identifying them in related researches? There are two potential reasons that educational seekers are not making up a large part of current MOOC participants:

1) While in most developing country, educational experience is often considered as the most valuable credential for a student to enter the job market. But most MOOCs cannot provide tangible educational assets to a student outside of the United States. Here is a quote from Alcorn's analysis of the current MOOC development in India: 
"Without trusted credentialing that can signal to an employer that a student has mastered the course content, the supply-chain from education to employment will break down and many students' primary motivation for enrollment will disappear" [7].

Even on a global level, only $18 \%$ of the MOOC students who seek educational benefits in MOOC believe they received tangible educational benefits out of it [20]. Since most of the educational benefit is intangible, MOOC students from developing countries, mainly India in this paper, are less likely to take MOOCs for a quality educational degree.

2) Another reason is the language barrier. Although there is XuetangX, the majority of the MOOCs today are still in English. But most developing countries have their local languages. Learning from English-based MOOCs requires these students to be competent in this second language [5]. Especially in China, where English is never the mother tongue, the majority of the student may not be able to understand the MOOC course content fully. It is difficult for a person without English skills even to become an educational seeker in MOOCs. This is also why Chinese MOOC industry has the most students with higher degrees: Only students with higher degrees are capable in English skills.

\section{Diverse Learner Behaviors}

Researchers have shown that students from different cultures behave differently in educational systems in many different ways, including off-task behaviors, help-seeking, and collaboration [21]. These differences are also significant in MOOC settings. In a study of an MOOC with 29,149 students from 172 countries, researchers found that students from countries with higher individualism and lower power distance, like The United States, are more likely to finish all course material (both lectures \& quizzes). In the meantime, students from countries with higher power distance and lower individualism, like China and India, focus more on evaluations and taking quizzes [21]. It also proves the notion that professional environment in Asian countries takes certificates more valuable than "soft knowledge."

As of MOOC completion status, dropout rate on MOOCs is substantially higher than traditional education in general [22]. But the three countries we exanimate also reflect a significant difference in MOOC completion rate. A study shows that non-U.S. students are more likely to receive certificates than U.S. students. For that particular analysis, certificate attainment for U.S. students is $3.72 \%$ on average, lower than attainment for Indian students at 5.12\% [23]. Going beyond geographic differences, these can be explained by cultural dimensions (power distance and individualism) and educational differences (discussion-centric or test-centric) [21]. Again, there are many factors associated with completion rate. It is unwise to only take certificate attainment to compare students' success in different countries. Stich found in her research that the intent to complete the course at the beginning highly correlates to the certificate attainment [6].

Another special learner behavior is that smartphone is becoming the primary learning device for MOOC students in China. For the first time, $82.25 \%$ of the Chinese student reported using mobile devices to access their MOOCs on a regular basis, Followed by desktop devices at $78.07 \%$, and tablet at $24.71 \%$ [12]. Although mobile MOOC market is steadily growing in the United States and India, there is currently no statistic on mobile usage for MOOC for these countries. While this mobile-centric MOOC environment in China is unique and interesting, it is not surprising given that China is leading the mobile first world on mobile payment and mobile commerce [24]. In China, 92\% of online users own a smartphone. But that drops to only $72 \%$ in the U.S. [25].

In India, offline access to MOOCs has become an effective solution to address the problem that around $66 \%$ of the Indian population does not have reliable access to broadband Internet and a computer [26]. According to Alcorn, limited access to Internet and computers and significantly weaker English skill make it impossible for rural Indians to connect to MOOCs [7]. So organizations are taking the initiative to help those Indians to access MOOC materials offline. An India company, Jaaga, combines MOOCs from different online sources, including Udacity, Stanford, Harvard, and MIT, to manage classes offline [27]. Companies like this not only provide video lectures to students, also they offer classroom seminars and individual instructions that help ESL 
(English as Second Language) students navigate through the courses [7]. This particular way of utilizing MOOC also result in a unique behavior pattern of some Indian MOOC students, these students tend to use the MOOC solely as a certification system and only complete certification test online [20].

\section{Branching Future}

From the previous sections, we identified and analyzed the general environment and special phenomenon exists in the MOOC industry in the U.S., China, and India. As different as they are already, will the industry develop differently in the future? The short answer is yes. All three countries may need to focus on addressing the uneven distribution of the population in MOOC enrollment, including broadens MOOC access for students of color in the U.S., for working professionals in China, and for women in India. Moreover, each county has its direction in developing MOOCs custom to their specialties. Many of the U.S. MOOC providers are pushing towards business-to-business MOOCs [28]. Major Chinese MOOC providers claim that 2016 is when "the area of paid online education is here" [12]. MOOC providers that target towards Indian market are working on validating their course certificates with leading Indian firms to appeal to more Indian students [13].

For those underrepresented populations in all three MOOC industries, we might be able to observe a trickle-down effect soon. The trickle-down effect is an economic phenomenon, which states that the process of a product adoption is from the "upper classes" to the "lower classes" within a society. Many studies indicate that despite the recent increase in enrollment in traditional higher education for underrepresented populations (students of color in the U.S. and female in India), their enrollment in general online education, including MOOCs do not follow the pattern [7,29]. At the 5th year of MOOC culture, the current users of MOOCs can still be the early adopter of the concept in their society. They are educated and white working professionals in the U.S., motivated and young students in China, and brilliant and male technicians or engineer in India. This groups not only have access to the newest ideas in the world, but they also have the time and ability to try them out. However, the increase in minority enrollment in the traditional higher education indicates the necessity of follow-up change in the current MOOC demographic. It is likely for us to see MOOCs provide greater access to minorities in the near future.

For future development, several U.S. MOOC providers are showing interest in the Business-toBusiness (B2B) MOOC market. As so many working professionals already showed interested in learning and developing through MOOCs, companies like Coursera identified corporate training as an enormous potential market for the industry. In 2016, Coursera announced "Coursera for Business." Udacity started to offer "Nanodegrees" and partner with Google, IBM, Mercedes-Benz, and several other industry leaders to provide training for future jobs. MOOC providers expect to see more users and user engagement with these programs since the businesses are promoting MOOCs to their employees. Companies from different industries are using these products to "arming their workforce with the in-demand skills of tomorrow" [30].

In China, MOOC provider believed that paid MOOC material is becoming the topic of the future. A survey shows that $70 \%$ of the MOOC students reported paying for some materials in MOOC in 2016, and the number was only $26 \%$ in $2015.41 .97 \%$ of them paid for video lectures, $26.29 \%$ paid for downloading study materials, and $18.11 \%$ paid for downloading mobile applications. Over half of the respondent said they would pay for materials that will help on their exams, study, or jobs [12]. Paying for valuable information is becoming a common sense among Chinese learners.

Development of MOOCs in India is more cater to link the MOOCs with the job market. Coursera executive Kabir Chadha is persuading leading Indian employers to validates certain MOOC completion certificates as a prerequisite of a candidate screening process [13]. This will motivate more Indian students to study MOOCs as the certificates start to have a signaling value to other employers as well. "For aspiring Indian engineers and scientists, online credentials offer a way to stand out from the crowd" [13]. Furthermore, this will create a close circle in bringing qualified professionals to the market. 


\section{Conclusions}

In conclusions, MOOCs is not borderless. It is different in the U.S., China, and India from various ways. Students in the United States are older, employed, and educated. Their main reason for taking MOOCs is for curiosity and fun. They are less likely to complete a course as they are less eager to be certified comparing to their Chinese and Indian counterparts. MOOC students from China are young and highly educated. Most of them are still students in traditional schools. They choose to take MOOCs to develop professional skills. They like to use mobile devices to access their MOOCs. Just as Indian students, Chinese students are more likely to complete tasks and exams instead of video lectures and other study material. In India, the majority of the MOOC students are recent graduates in the technology industry, and they took MOOC to stand out from their competitors in the job market. Most of them are male and, again, highly educated. In recognition of these differences, we understand that MOOCs is not a "one-size-fits-all" product. To address the issues appeared in different countries and to improve the MOOC industry as a whole, distinct steps that cater to different demands need to be taken. In the future, MOOCs will also walk on a different path in the United States, China, and India.

\section{References}

[1] Dave Cormier and George Siemens, "Through the Open Door: Open Courses as Research, Learning, and Engagement," EDUCAUSE Review, vol. 45, no. 4, pp. 30-39, July/August 2010.

[2] Pappano Laura. (2012, November) The New York Times. [Online]. HYPERLINK "http://www.nytimes.com/2012/11/04/education/edlife/massive-open-online-courses-aremultiplying-at-a-rapid-pace.html?pagewanted=all\&_r=0"

[3] Dhawal Shah. (2016, December) MOOC List. [Online]. HYPERLINK "https://www.classcentral.com/report/moocs-stats-and-trends-2016/"

[4] Gayle Christensen et al., "The MOOC Phenomenon: Who Takes Massive Open Online Courses and Why?," November 2013.

[5] T. Liyanagunawardena, Williams S., and Adams A, The impact and reach of MOOCs: a developing countries' perspective.: eLearning Papers, 2013, vol. 33.

[6] Amy E. Stich and Todd D. Reeves, "Massive open online courses and underserved students in the United States," Internet and Higher Education, vol. 32, pp. 58-71, January 2017.

[7] Brandon Alcorn, Gayle Christensen, and Devesh Kapur, Higher Education and MOOCs in India and the Global South, Change: The Magazine of Higher Learning., 2015, vol. 47, DOI: 10.1080/00091383.2015.1040710.

[8] MOOCs University. (2017) http://www.moocsuniversity.org/. [Online]. HYPERLINK "http://www.moocsuniversity.org/"

[9] United States Census Bureau. (2017, June) United States Census Bureau. [Online]. HYPERLINK "https://www.census.gov/popclock/"

[10] Dhawal Shah. (2016, December) Class Central. [Online]. HYPERLINK "https://www.classcentral.com/report/coursera-2016-review/"

[11] Dhawal Shah. (2016, October) Class Central. [Online]. HYPERLINK "https://www.classcentral.com/report/xuetangx/"

[12] Guokr MOOC \& icourse163, "2016 Knowledgeable Youth Report," 2017.

[13] George Anders. (2015, July) MIT Technology Review. [Online]. HYPERLINK "https://www.technologyreview.com/s/539131/india-loves-moocs/" 
[14] Karen R. Humes, Nicholas A. Jones, and Roberto R. Ramirez, "Overview of Race and Hispanic Origin: 2010," 2011.

[15] National Bureau of Statistics of the People's Republic of China, "2015 National 1\% Population Sampling Survey Bulletin," Beijing, 2016.

[16] Office of the Registrar General \& Census Commissioner, India, "2011 Census Data," Ministry of Home Affairs, Government of India, 2012. [Online].

HYPERLINK "http://www.censusindia.gov.in/2011-Common/CensusData2011.html"

[17] Archana Renju, "Higher Education and Women Participation in India," GLOBAL JOURNAL FOR RESEARCH ANALYSIS, vol. 3, no. 6, June 2014.

[18] Lori Breslow et al., "Studying Learning in the Worldwide Classroom Research into edX's First MOOC," Research \& Practice in Assessment, vol. 8, pp. 13-25, 2013.

[19] John Covach. (2016, January) The Conversation. [Online]. HYPERLINK "http://theconversation.com/confessions-of-a-mooc-professor-three-things-i-learned-and-twothings-i-worry-about-53330"

[20] Chen Zhenghao et al. (2015, September) Harvard Business Review. [Online]. HYPERLINK "https://hbr.org/2015/09/whos-benefiting-from-moocs-and-why"

[21] Zhongxiu Liu et al., "MOOC Learner Behaviors by Country and Culture; an Exploratory Analysis," 2016.

[22] Doug Clow, "MOOCs and the funnel of participation," Third Conference on Learning Analytics and Knowledge (LAK 2013), no. 8-12, pp. 185-189, April 2013.

[23] Sergiy O. Nesterko et al., "Evaluating Geographic Data in MOOCs," in the 2013 conf. on Neural Information Processing Systems, 2013.

[24] Michelle Evans. (2017, April) Forbes. [Online]. HYPERLINK "https://www.forbes.com/sites/michelleevans1/2017/04/12/how-china-won-the-race-to-beingconsidered-a-mobile-first-commerce-nation/"

[25] Stewart Rogers. (2016, February) Venture Beat. [Online]. HYPERLINK "https://venturebeat.com/2016/02/12/new-data-shows-the-staggering-growth-of-apps-andsmartphones-in-china/"

[26] Internet World Stats. (2017, March) Asia \& North America. [Online]. HYPERLINK "http://www.internetworldstats.com/asia/in.htm"

[27] Faizul Nisha, "MOOCs: Changing Trend Towards Open Distance Learning with Special Reference to India," DESIDOC Journal of Library \& Information Technology, vol. 35, no. 2, pp. 82-89, April 2015.

[28] Dhawal Shah. (2016, December) Class Central. [Online]. HYPERLINK "https://www.classcentral.com/report/biggest-mooc-trends-2016/"

[29] J. Herrera, D. Jones-Davis, A.Q. Gates, S.S. Jaggars, and M. Suiter, "Online learning: An opportunity for minority serving institutions?," Society for the Advancement of Chicanos/Hispanics and Native Americans in Science, January 2014.

[30] (2016) Coursera. [Online]. HYPERLINK "https://www.coursera.org/enterprise"

[31] Amit Kumar Das, Anamika Das, and Soumit Das, "Present Status of Massive Open Online Course (MOOC) initiatives for Open Education Systems in India - An Analytical Study," Asian Journal of Multidisciplinary Studies, vol. 3, no. 7, July 2015. 